\title{
Index to genera and species, Volume 5 (1994)
}

\author{
Acari \\ Ameronothrus dubinini Sitnikova 177, 180, 184 \\ Berniniella bicarinata (Paoli) 214 \\ - sigma (Strenzke) 214 \\ Camisia horrida (Hermann) 184 \\ Ceratoppia bipilis (Hermann) 177, 180, 181, 184 \\ Chamobates spinosus Sellnick 213, 215 \\ - voigtsi (Oudemans) 216 \\ Diapterobates humeralis (Hermann) 177, 180-181, 184 \\ Gymnodamaeus bicostatus (C.L. Koch) 213-214 \\ Microzetorchestes emeryi (Goggi) 213-214 \\ Moritzoppia keilbachi (Moritz) 214-215 \\ - tridentata (Forsslund) 215 \\ - unicarinata (Paoli) 215 \\ Nothrus invenusta (Michael) 213 \\ Odontocepheus elongatus (Michael) 213-214 \\ Oliqonychus ununguis Jacob 182 \\ Oppiella minidentata (Subias) 213 \\ - minidentata (Subias) 215 \\ Oribatella calcarata Koch 184 \\ - sexdentata Berlese 213,216 \\ Quadroppia michaeli Mahunka 213, 215 \\ - quadricarinata (Michael) 215 \\ Scutovertex sculptus Michael 215 \\ Trypochthonius sp. 184 \\ Xenillus latus (Nicolet) 213-214 \\ Xylobates capucinus (Berlese) 213, 215
}

\section{Coleoptera}

Further 59 taxa are listed on pp. 162-163, and many of them discussed on pp. 157-167.

Agabus 169-170, 174

- adpressus Aubé 169-171

- adpressus-group 169-171

- angusi Nilsson sp. n. 169, 172-174

- approximatus Fall 172

- chalconatus-group 170

- clypealis (Thomson) 169, 173, 174

- confinis-group 169,172, 173

- congener (Thunberg) 174

- discolor (Harris) 169, 172-174

- erichsoni-group 170

- haeffneri Aubé 170

- levanderi Hellén 169, 172-174

- mimmi J. Sahlberg 170

- opacus Aubé 170
- opacus-group 170

- sahlbergi Sharp 170

- solus Leech 170

- udege Nilsson sp. n. 169-173

- wasastjernae C. R. Sahlberg 170

Agrilus pseudocyaneus Kiesenwetter 2 Anacaena lutescens (Stephens) 55 Atomaria pseudaffinis Johnson \& Strand 6 Berosus fulvus Kuwert 55

Cercyon borealis Baranowski 55

- emarginatus Baranowski 55

Ceutorhynchus pollinarius (Forster) 6

Clambus 1

- nigrellus Reitter 1, 2

Coccidula scutellata (Herbst) 1

Colymbetes subquadratus Motschulsky 170

Cossonus cylindricus Sahlberg 2

Cyllodes ater (Herbst) 203-204

Cyphea latiuscula Sjöberg 6

Dianous coerulescens (Gyllenhal) 1

Donacia tomentosa Ahrens 6

Eucnemis capucina Ahrens 2

Gaurodytes adpressus (Aubé) 170

- angusticollis J. Sahlberg 170

- discolor (Harris) 172

Gnypeta ripicola (Kiesenwetter) 1

Gyrophaena 39

- affinis Mannerheim 39, 40

- kangasi Rutanen sp. n. 39, 40

- obsoleta Ganglbauer 39,40

- rosskotheni Wüsthoff 39,40

- rousi Dvorak 39,40

Haenydra 53

Hydraena 53, 54

- belgica d'Orchymont 53

- britteni Joy 54

- excisa Ganglbauer 53-55

- gracilis Germar 53-55

Hydrochus megaphallus Berge Henegouwen 55

Hydrovatus 103

- asymmetricus Biström \& Wewalka sp. n. 103, 104

- turbinatus Zimmermann 104

Hypera viciae (Gyllenhal) 1

Ilybius 170

Magdalis 5

- ruficornis (L.) 5

Malthodes 2

- fibulatus Kiesenwetter 2

- maurus (Laporte de Castelnau) 2

- misellus Kiesenwetter 2 
Mycetochara flavipes (Fabricius) 201

Mycetophagus fulvicollis Fabricius 201

Nargus badius (Sturm) 2, 3

- velox (Spence) 2, 3

Ochthebius kaninensis Poppius 55

- nilssoni Hebauer 55

- rugulosus Wollaston 55

Oryctes nasicornis (L.) 3

Paromalus parallelepipedus (Herbst) 201

Philhygra obtusangula Joy 1

Ptilinus fuscus Geoffrey 201

Ptomaphagus sericatus (Chaudoir) 3

- subvillosus (Goeze) 3

Scolytus 5

Sphaeridium marginatum Fabricius 55

- substriatum Faldermann 55

Thinodromus arcuatus (Stephens) 1

Trichonyx sulcicollis (Reichenbach) 3

Triplax rufipes (Fabricius) 201

Xyletinus tremulicola Y. Kangas 201

\section{Collembola}

Anurophorus septentrionalis Palissa 185

Entomobrya nivalis (Linnaeus) 185

\section{Diptera}

Botanophila silvatica (Robineau-Desvoidy) 186

Bradysia 42

- spinipennis Sasakawa 41, 42, 48

Chromatomyia linnaeae Griffiths 187-188

Ctenosciara 42

Dialineura anilis (Linnaeus) 186

Dinempheria 13, 14, 16

- enigmata Väisänen sp.n. 13-15

Dolichosciara 41-43

Drosophila buzzatii Patterson \& Wheeler 149

- hydei Sturtevant 149

- mercatorum Patterson \& Wheeler 149

- repleta Wollaston 149,150

Echinopodium 16

Exechia separata Lundström 186

Exechia sp. 186

Lobosciara 41-43

- adebratti Vilkamaa \& Hippa sp. n. 41, 43, 45-47

- bilobata Vilkamaa \& Hippa sp. n. 41, 43, 44, 46, 47

- latiloba Vilkamaa \& Hippa sp. n. 41, 43, 44, 47, 48

- spinipennis (Sasakawa) 41, 43,46-48

- trilobata Vilkamaa \& Hippa sp. n. 41-43, 45, 47, 48

Moriniola 13

Mycoleia 16

Mycomya $13,14,16,18,19$

- affinis (Staeger) 20

- edra Väisänen sp. n. 13, 20, 21

- lightfooti Edwards 13, 18, 20
- londti Väisänen sp. n. 13, 18-20, 22

- malkini Väisänen 20

- marginata-group 18-20

- natalensis Väisänen sp. n. 13, 16-19

- trilineata-group 20

Mycoya (Mycomyopsis) 20

Mycomyiella 13,14

- camerouensis Matile 22

- irwini Väisänen sp. n. 13, 21, 22

- sericulata Matile 22

Neoempheria 13, 14, 16, 24

- ornata Okada 16

- transvaalensis Väisänen sp. n. 13, 22-24

Parempheriella 13

- australis Väisänen sp. n. 13, 25, 26

- flava (Matile) 26

- isoseta-group 26

Pegohylemyia sp. 186

Pegomya geniculata (Bouché) 186

- maculata Stein 186

Pegonya sp. 186

Phytomyza periclymeni DeMeijere 188

Phytosciara 42

Prosciara 42, 147

- didactyla Kjærandsen sp. n. 147, 148

- megachaeta Hippa \& Vilkamaa 147, 148

Scatopsciara 42

Suillia atricornis (Meigen) 186

- bicolor (Zetterstedt) 186

Syndocosia 13

Tarnania fenestralis (Meigen) 186

Thereva nobilitata (Fabricius) 186

Tipula 49

Tipula (Tipula) 49,50

- czizeki de Jong 49, 50

- fimbriata Meigen 49

- oleracea L. 49-52

- paludosa Meigen 49-52

- subcunctans Alexander 49-52

Viridivora 13

Xenopygina 42

\section{Heteroptera}

Further 59 entries from Iraq listed on pp. 87-95.

Acrosternum millierei (Mulsant \& Rey) 155

Aethus perithrix Mancini 154

- pilosulus (Klug) 155

Anepsiocoris encaustus (Puton) 155

Atomoscelis brevicornis Reuter 211

Atractotomus magnicornis (Fallén) 184

Camptocoris typus (Diatant) 155

Coranus angulatus Stål 155

Coridius viduatus (Fabricius) 155

Cyrtopeltis (Campyloneuropsis) pygmaea Wagner 211

Cyrtopelits khalafi Seidenstücker 212 
Dieuches forbesi (Kirkaldy) 155

Elasmotethus brevis Lindberg 6

Ellenia Reuter 211

Emblethis gracilicornis Puton 155

Geocoris socotranus Kirkaldy 155

Lygus rugulipennis Poppius 184

Macroscytus brunneus (Fabricius) 155

Moissonia Reuter 211

Nariscus spinosus (Burmeister) 155

Orthops rubricatus (Fallén) 184

Pachybrachius dubius (Reuter) 155

Peritropis armillarius Schmitz 151, 152

- maculicornis Linnavuori \& Al-Safadi 151, 152

- selene Linnavuori sp. n. 151, 152, 155

Phaenotropis cleopatra (Horváth) 155

Piezodorus rubrofasciatus (Fabricius) 155

Pirates strepitans Rambur 155

Scantius forsteri (Fabricius) 155

Serranegra petrophila Lindberg 155

Tarajala Wagner 211

Tenosius proletarius (Schaum) 155

Tuponia (Chlorotuponia) brevicornis (Reuter) 211

- (Chlorotuponia) filomele Linnavuori sp. n. 209-211

- (Chlorotuponia) guttata Wagner 208, 209, 210

Tuponia altera Wagner 208, 209

- annulata Wagner 205

- bifasciata Wagner 208

- bilobata Benedek \& Jászai 208

- canariensis Wagner 209-211

- carayoni vulnerata Linnavuori 205, 206

- dentifer Wagner 206

- elegans (Jakovlev) 208, 209

- macedonica Wagner 206, 207

- oculata Wagner 209-211

- punctipes Reuter 211

- pungens Linnavuori 207

- roseipennis Reuter 207

- semele Linnavuori sp. n. 206, 208

- sodomita Wagner 209

Yotvata (Godataira) aqualla Linnavuori 154

- ectagela Linnavuori 154

- hadibo Linnavuori sp. n. 151, 153-155

- hawtana Linnavuori 154

- pulchella Linnavuori 154

- pulcherrima Linnavuori 154

- Salmana Linnavuori 154

Yotvata (Yotvata) albocunealis Linnavuori 153

- erifyle Linnavuori sp. n. 151-153. 155

- nergal Linnavuori 153

- picticornis (Horváth) 153

\section{Homoptera}

\section{Adelges sp. 184}

Aphis epilobiaria Thunberg 4

- fabae (Scopoli) 110

- fabae cirsiiacanthoides (Scopoli) 110, 111
- idaei van der Goot 111, 113

- klimeschi (Börner) 3, 4, 111

- leontodontis (Börner) 3,4

- molluginis (Börner) 4

- violae Schouteden 3,4

Aulacorthum 111

Balclutha punctata (Fabricius) 5

Chaitophorus populeti (Panzer) 112

- populialbae (Boyer de Fonscolombe) 4, 5, 112

- tremulae Koch 4

Cinara sp. 184

Elatobium abietinum (Walker) 184

Empoasca vitis (Göthe) 5

Geoica setulosa (Passerini) 112

Glyphina ?pseudoschrankiana Blackman 110, 112

Macrosiphoniella 111

- absinthii (Linnaeus) 110

- artemisiae (Boyer de Fonscolombe) 110

- millefolii (DeGeer) 110

Metopeurum fuscoviride Stroyan 111

Peuceptyelus coriaceus (Fallén) 5

Phenacoccus piceae (Löw) 184

Pterocomma pilosum konoi Hori ex Takahashi 110

- salicis Linnaeus 110

Uroleucon obscurum (Koch) 5

- pilosellae (Börner) 5

- tanaceti (Linnaeus) 111

Zygina rosincola (Cerutti) 5

\section{Hymenoptera}

Further 20 Atractodes species and their synonymies are listed on pp. 129-133.

Ablaxia squamifera (Thomson) 5

Aleiodes gastritor (Thunberg) 28, 30, 31, 33

Amauronematus 135

- compactus Bogacheva 135, 136

- harpicola Bogacheva 135, 137, 138

Aniseres 117

- lapponicus Jussila sp. n. 115, 117, 119, 121

- pallipes Förster 115-117, 119, 121

- paradoxus van Rossem 117

Apanteles 28-31, 33

Aperileptus 116, 120

- microspilus Förster 119

- rossemi Jussila sp. n. 115, 116, 119, 120

- spoliator Förster 119

- viduatus Förster 119, 120

Atractodes 129

Bassus minutissimus Zetterstedt 123

Caenolyda reticulata (L.) 6

Campoletis varians (Thomson) 30

Cotesia jucunda (Marshall) 28, 30

- melanoscela (Ratzeburg) 32

Dacnusa 8

Dacnusa aterrima Thomson 8 
- bakurianensis Gannota \& Tobias sp. n. ..7-9

- cubocephala $<$ sic! = bakurianensis $>8$

Dicolus pectoralis Förster 119

- subtiliventris Förster 119

Entypoma 121

- robustator Aubert 119, 121

Eulophus larvarum (L.) 29-31

Helictes 121

- fabularis van Rossem 119, 121

- incongruens van Rossem 119, 121

Itamoplex armator (Villers) 33

Formica pratensis Retzius 4

Lasius niger (L.) 3

Megastylus pectoralis (Förster) 119

Mesoleptus laevigatus (Gravenhorst) 129

Myrmica rubra L. 4

- sabuleti Meinert 139

Orthocentrus 122

- ambiguus Holmgren 119, 122

- frontator (Zetterstedt) 122

- hirsutor Aubert 119, 122

- laticollis Holmgren 123

- marginatus Holmgren 122

- patulus Holmgren 119, 122

- petiolaris Thomson 122

- thomsoni Roman 119, 122

Oxytorus luridor (Gravenhorst) 129

Phobocambe neglecta (Holmgren) 28, 30, 33

Plectiscidea 120

- nava (Förster) 119, 120

- substantiva van Rossem 119, 120

- subteres (Thomson) 115, 116, 119-121

- sustantiva <sic! > van Rossem 120

Plectiscus subteres Thomson 116, 120

Proclitus navus Förster 120

Protapanteles immunis (Haliday) 28, 30

Sinophorus turionus (Ratzeburg) 29, 30

Stenomacrus laticollis (Holmgren) 119, 123

- minutissimus (Zetterstedt) 119, 123

Stilpnus tenebricosus (Gravenhorst) 129

Symplecis breviuscula Roman 119, 122

- infavorabilis van Rossem 122

- invisitata van Rossem 115, 117, 119, 121, 122

Zele deceptor Wesmael 29-31

\section{Lepidoptera}

Further 592 entries from Kola Peninsula listed on pp. 6583.

Callimorpha dominula (L.) 6

Calliteara pudipunda (L.) 6

Cusruotes freyerella (Hübner) 97, 102

Depressaria chaerophylli Zeller 6

Elachista 35

- adelpha Kaila \& Jalava sp. n. 97-99

- albifrontella (Hübner) 35

- apicipunctella Stainton 35
- argentella Clerck 100

- bedellella Sircom 100, 101

- bifasciella Treitschke 98

- bifasciella-group 35

- coeneni Traugott-Olsen 100, 101

- coeneni titanella Kaila \& Jalava ssp. n. 97, 100-102

- dimicatella Rebel 98

- elegans Frey 98

- fuscofrontella Sruoga 35-37

- habeleri Traugott-Olsen 100

- nobilella Zeller 35

Endothenia gentianaeana (Hübner) 189192, 195-196

- marginana (Howarth) 189195

- marginana tarandina Laasonen \& Laasonen ssp. n. 189, 191-195

- oblongana (Howarth) 189192, 195

Epirrita autumnata (Borkhausen) 27-33

Gnorimoschema 57, 58, 60-62

- antiquum Povolný 62

- antiquum montanum Povolný 57,62

- batanella (Busck) 60

- bodillum Karlsholt \& Nielsen 62

- epithymellum (Staudinger) 57,61

- epithymellum kirgisicum Povolný ssp. n. 57, 59,61,62

- herbichi (Nowicki) 62

- herbichi kamchaticum Povolný 57,62

- jalavai Povolný sp. n. 57-60

- mikkolai Povolný sp. n. 57, 58,60,61

- nordlandicolellum (Strand) 57, 59, 62, 63

- radkevichi Piskunov 60

- streliciella (Herrich-Schäffer) 62

- subterraneum Busck 61

Gobipalpa 57

- inexpectata Povolný 57, 59, 64

Lymantria dispar (L.) 32

Maculinea arion (L.) 139-145

Plutella interrupta (Walsingham) 200

- poulella (Busck) 200

Rhigognostis annulatella (Curtis) 199

- incarnatella (Steudel) 198-199

- japonica (Moriuti) 199

- kovacsi (Gozmany) 198-199

- kuusamoensis Kyrki 198-199

- schmaltzella (Zetterstedt) 198-199

- senilella (Zetterstedt) 199-200

- sibirica Kyrki 197-200

- wolfschlaegeri (Rebel) 199

Stephensia abbreviatella (Stainton) 97

Xestia 125

Xestia (Anomogyna) 125

- alpicola (Zetterstedt) 125, 126

- alpicola alpicola (Zetterstedt) 128

- alpicola camica (Hering) 128

- alpicola hyperborea (Zetterstedt) 128

- alpicola iveni (Hübner) 125, 128

- alpicola arctica $<$ sic! $>\rightarrow$ alpicola alpicola (Zetterstedt) 128

- speciosa (Hübner) 125-127

- speciosa arctica Zetterstedt 126, 127 
Xestia speciosa baltica (Valle) 125-127

- speciosa speciosa (Hübner) 126, 127

- viridescens (Turati) 125-127

\section{Mallophaga}

Anaticola boettikeri Eichler \& Vasjukova 6 - rubromaculatus (Rudow) 6

Carduiceps 5

- meinertzhageni Timmermann 5
Phalangida

Mitopus morio (Fabricius) 185

\section{Psocoptera}

Caecilius burmeisteri Brauer 184

- despaxi Badonnel 184

Stenopsocus lachlani Kolbe 184 